\title{
Efeitos de calagem e adubação no crescimento e nutrição de arnica $^{1}$
}

\author{
Antônio Claret de Oliveira Júnior; Valdemar Faquin; José Eduardo Brasil P Pinto \\ UFLA, C. Postal 3037, 37200-000 Lavras-MG; E-mail: antoniocoliveirajr@yahoo.com.br
}

\begin{abstract}
RESUMO
A arnica é uma planta amplamente usada na medicina popular que sofre risco de extinção. Este trabalho objetivou avaliar o crescimento, teor e acúmulo de nutrientes e alumínio na parte aérea de arnica em resposta à calagem e adubação. O experimento foi conduzido em casa de vegetação, em amostra de Cambissolo álico, em esquema fatorial $2 \times 3+1$, sendo dois níveis de calagem (sem e com), três tipos de adubação (mineral, orgânica e mista). Para controle foi utilizado um tratamento adicional, constituído de solo proveniente da região endêmica da arnica, para simular suas condições naturais de desenvolvimento. Foram utilizadas quatro repetições e uma planta por vaso. Tomaram-se as alturas inicial e final, obtendo-se o crescimento relativo (CR) e determinaram-se as massas secas de parte aérea (MSPA) e de raiz (MSR). Foram determinados o teor e o acúmulo de nutrientes e alumínio na MSPA. De modo geral, a arnica apresentou maiores valores MSPA, MSR e CR com adubos minerais e menores com orgânico. Para MSPA, a calagem foi essencial somente nos tratamentos que receberam adubo orgânico. A arnica é uma planta tolerante ao $\mathrm{Al}$, exigente em $\mathrm{Zn}$ e Mn e não exigente em macronutrientes.
\end{abstract}

Palavras-chave: nutrição mineral, planta medicinal, cultivo, Lychnophora pinaster.

\author{
ABSTRACT \\ Effects of liming and fertilization on growth and nutrition of \\ arnica
}

Arnica is a plant widely used in folk medicine, which is in danger of extinction. This work aimed to evaluate growth, nutrients and aluminum contents and quantity in aboveground dry matter under liming and fertilization. The experiment was carried out in greenhouse, with allic Cambisol samples in 2x3+1 factorial scheme, using two liming levels (with and without) and three fertilization types (mineral, organic and mixed). Control was an additional treatment, using soil from arnica endemic land, to simulate its natural conditions of development. Four replications were used and one plant for each pot. Relative growth (RG) was obtained from initial and final heights. Aboveground dry matter (ADM) and the root dry matter (RDM) were determined. The contents and accumulation of nutrients and aluminum were determined in ADM. Generally, arnica showed higher ADM, RDM and RG with mineral fertilization and lower ADM, RDM and RG with organic fertilization. For ADM, liming was essential only in treatments with organic fertilizer. Arnica is not Al-tolerant, it is $\mathrm{Zn}$ - and Mn-exigent, but is not macronutrientsexigent plant.

Keywords: Mineral nutrition, medicinal plant, cropping, Lychnophora pinaster.

\section{(Recebido para publicação em 19 de setembro de 2005; aceito em 27 de julho de 2006)}

$\mathrm{A}^{\circ}$ o longo do tempo, a farmacopéia popular consagrou diversas plantas como medicamentos naturais. No entanto, apesar da alta demanda, ainda é relativamente pequeno o conhecimento acumulado sobre o cultivo das espécies medicinais nativas do Brasil. Isso tem acarretado grande devastação dos recursos da flora nacional que, além dos danos ambientais, gera dificuldades no controle de qualidade dos fitofármacos.

Considerado endêmico dos campos rupestres do Cerrado brasileiro, o gênero Lychnophora apresenta dezenas de espécies, todas com microendemismo bastante pronunciado (Semir, 1991). Devido ao seu amplo uso na medicina popular, a Lychnophora ericoides, junto das demais espécies do gênero, vêm sofrendo grande pressão de extrativismo, sendo consideradas espé- cies vulneráveis à extinção (Sociedade Botânica do Brasil, 1992). Essa situação sugere que as espécies encontradas nos campos rupestres são merecedoras de grande prioridade de conservação, visto que essas plantas compõem flora especializada, de distribuição geográfica muito restrita (Oliveira-Filho; \& Fluminhan-Filho, 1999). Nesse sentido, Silva (1994) afirma que, se formas de sobrevivência para Lychnophora pinaster não forem asseguradas, podese chegar à sua extinção.

Pelo fato do interesse no cultivo comercial de plantas medicinais ser recente, informações sobre o crescimento e nutrição dessas espécies ainda são escassas e dispersas, sobretudo para as originadas do Cerrado. Os poucos trabalhos científicos encontrados sobre arnica fazem referência a estudos florísticos (Carvalho, 1992), fenológicos (Silva, 1994), fitoquímicos (Pinheiro, 2002), de micropropagação (Souza, 2003), conservação de germoplasma (Paron et al., 2005) e produção de óleo essencial (Oliveira Júnior et al., 2005).

O cultivo em nível comercial da arnica pode ser capaz de fornecer material vegetal de qualidade para a indústria farmacêutica, auxiliando, inclusive, na solução do problema de extrativismo que essa espécie sofre (Souza, 2003). Dessa forma, estudos a respeito do crescimento de arnica em condições de cultivo ganham destaque, pois podem, inclusive, auxiliar no estabelecimento de coleções de conservação de germoplasma, conforme sugerido por Paron et al. (2005).

O presente trabalho teve como objetivo avaliar o crescimento, o teor e

${ }^{1}$ Parte da dissertação do primeiro autor, apresentada ao Curso de Pós-graduação em Solos e Nutrição de Plantas da Universidade Federal de Lavras, para obtenção do título de Mestre. 
acúmulo de nutrientes e alumínio na parte aérea de mudas de arnica (Lychnophora pinaster) em resposta às aplicações de calcário e de adubos mineral e orgânico.

\section{MATERIAL E MÉTODOS}

O experimento foi conduzido em casa de vegetação do Departamento de Ciência do Solo da Universidade Federal de Lavras (UFLA), em amostra de Cambissolo álico, coletada na profundidade de $0-20 \mathrm{~cm}$, em vasos de $3 \mathrm{dm}^{3}$. Após destorroamento, a amostra foi seca ao ar e peneirada em malha de $4 \mathrm{~mm}$. Foram tomadas subamostras para caracterizações físicas e químicas (Tabela 1), conforme Embrapa (1997) e do P-remanescente de acordo com Alvarez V. et al. (2000).

Os tratamentos foram constituídos pelo uso ou não de calagem, três tipos de adubação (mineral, orgânica e mista - 50\% de cada) e um tratamento adicional como controle, simulando as condições naturais de crescimento da arnica. Os tratamentos foram arranjados em esquema fatorial $2 \times 3+1$, no delineamento experimental inteiramente casualizado, com quatro repetições.

Utilizou-se calcário dolomítico calcinado, micropulverizado, com 35\% de $\mathrm{CaO}, 14 \%$ de $\mathrm{MgO}$ e PRNT $=100 \%$, para elevar a saturação de bases do solo para $50 \%$, equivalente a uma dosagem de 1,3 $\mathrm{t} \mathrm{ha}^{-1}$. A adubação mineral, baseada em Novais et al. (1991), constituiu-se de $\left(\mathrm{mg} \mathrm{dm}^{-3}\right.$ de solo) $\mathrm{N}=150$; $\mathrm{P}=200 ; \mathrm{K}=150 ; \mathrm{S}-\mathrm{SO}_{4}=40 ; \mathrm{B}=0,5$; $\mathrm{Cu}=0,8$ e $\mathrm{Zn}=3,00$. As doses de $\mathrm{N}$ e $\mathrm{K}$ foram parceladas em três vezes (plantio, 30 e 60 dias). Como adubo orgânico foi utilizado o esterco de curral curtido, seco a $65^{\circ} \mathrm{C}$, moído e passado em peneira de $1 \mathrm{~mm}^{2}$. A dose foi baseada em CFSEMG (1999), adotando-se $32 \mathrm{t}$ $\mathrm{ha}^{-1}$, dose essa equivalente a dez por cento do volume de solo, nos vasos dos tratamentos essencialmente orgânicos. A análise química do esterco de curral (Tabela 2) foi realizada conforme Tedesco et al. (1995). Os tratamentos com adubação mista receberam metade das doses dos tratamentos individuais (50\% mineral e 50\% orgânica). Após a aplicação dos tratamentos o solo foi in- cubado por 40 dias, com umidade de $50 \%$ do volume total de poros (VTP), quando foram amostrados para novas análises químicas (Tabela 1).

O tratamento controle foi composto de amostras da camada de 0-20 cm de profundidade de um Neossolo Litólico, coletado em Lavras-MG, na Serra da Bocaina (área endêmica da arnica), com o intuito de simular as condições naturais da arnica. Esse material também foi peneirado, incubado pelo mesmo período, porém sem aplicação de qualquer tratamento de melhoria da fertilidade. Sua composição física e química está apresentada na Tabela 1 .

Os tratamentos que receberam somente adubação mineral e aquele pertencente ao grupo controle tiveram vasos preenchidos com 2,6 L de solo. Os demais vasos foram preenchidos com 2,86 L (2,6 L de solo + 10\% de esterco) para adubação orgânica e 2,73 L (2,6 L+5\%) para adubação mista.

As mudas de arnica foram produzidas no Laboratório de Biotecnologia da UFLA, utilizando segmentos nodais, obtidos por meio de germinação de embriões in vitro, com indução de multiplicação em meio de cultura. Depois de permanecerem na câmara de crescimento, foram aclimatadas durante 30 dias em casa de vegetação, em potes plásticos preenchidos com material do Neossolo Litólico, como descrito em Souza (2003). Após a aclimatação uma única muda foi transplantada, com torrão, para os vasos contendo os tratamentos, onde foram cultivadas por 150 dias. A umidade no solo foi mantida em 50\% do VTP, por meio de irrigações periódicas com água deionizada.

Durante o período de experimento, foram tomadas medidas das alturas inicial e final das plantas de cada parcela, para estimar o seu crescimento relativo (CR); através da equação: $\mathrm{CR}(\%)=$ 100x[(altura final-altura inicial)/altura inicial]. Na colheita, as plantas foram cortadas rente ao solo. Descartou-se a parte lignificada do caule e o restante foi seco em estufa de circulação de ar forçada a $65-70^{\circ} \mathrm{C}$ e pesadas para obtenção da matéria seca da parte aérea (MSPA). Em seguida, a MSPA foi moída em moinho do tipo Willey e analisada quanto aos teores de nutrientes e Al, sendo este último determinado através do método de colorimetria do aluminon (Malavolta et al., 1997). As raízes foram retiradas através de destorroamento e peneiramento do solo seco, determinando-se a massa seca de raízes (MSR).

As variáveis de crescimento (MSPA, MSR e CR) foram submetidas à análise de variância e os tratamentos comparados pelo teste de Scott-Knott $(\mathrm{p}<0,05)$. Adicionalmente, foram aplicadas análises de correlações lineares entre as variáveis de crescimento e os parâmetros de fertilidade dos solos e elementos (nutrientes e alumínio) no tecido vegetal. A significância dos valores de correlação foi comparada pelo teste de tstudent (1 e 5\%).

\section{RESULTADOS E DISCUSSÃO}

Os tratamentos influenciaram significativamente as variáveis de crescimento das plantas de arnica. As produções de MSPA, MSR e CR foram influenciadas pela interação entre calagem e adubação (Tabela 3). Provavelmente, o efeito da adubação na MSPA, independente da presença de calcário, tenha sido devido à pronta disponibilidade de nutrientes, fornecidos via adubos minerais. Para os tratamentos com adubação orgânica, a aplicação de calcário promoveu maior produção de MSPA, possivelmente, devido à elevação do $\mathrm{pH}$ (Tabela 1) e conseqüente aumento na atividade microbiológica e mineralização do esterco. Pode ter sido disponibilizado mais enxofre, uma vez que houve correlação $\left(r=0,78^{*}\right)$ entre o teor deste nutriente no solo com a MSPA. O teor de $\mathrm{S}_{-} \mathrm{SO}_{4}$ no solo sob adubação orgânica com calagem foi cerca de $16 \%$ maior que no solo sem calagem e mais próximo do controle (Tabela 1).

De modo geral, a calagem não afetou a MSR, ou seja, não houve diferenças entre os tratamentos em relação à aplicação de calcário, à exceção do tratamento mineral que teve MSR menor. Esses resultados demonstram que o crescimento radicular da arnica não é influenciado por acidez e alta saturação por $\mathrm{Al}(\mathrm{m} \%)$ do solo. De maneira geral, estas propriedades do solo afetam negativamente o crescimento das raízes da grande maioria das plantas de interesse 
Tabela 1. Composição química e física do Neossolo Litólico (Controle) e do Cambissolo álico na sua condição natural (CN) e após aplicação dos tratamentos. Lavras, UFLA, 2004.

\begin{tabular}{|c|c|c|c|c|c|c|c|c|}
\hline \multirow{3}{*}{ Atributo } & \multirow{3}{*}{ Controle } & \multirow{3}{*}{$\mathrm{CN}$} & \multicolumn{6}{|c|}{ Adubação } \\
\hline & & & Mineral & Orgânica & Mista & Mineral & Orgânica & Mista \\
\hline & & & \multicolumn{3}{|c|}{ Com calagem } & \multicolumn{3}{|c|}{ Sem calagem } \\
\hline $\mathrm{pH}$ & 4,7 & 5,3 & 5,7 & 6,2 & 5,9 & 5,3 & 5,5 & 5,3 \\
\hline$P\left(\mathrm{mg} \mathrm{dm}^{-3}\right)$ & 7,1 & 1,2 & 22,2 & 1,3 & 17,4 & 67,9 & 1,2 & 17,5 \\
\hline $\mathrm{K}\left(\mathrm{mg} \mathrm{dm}^{-3}\right)$ & 41,0 & 17,0 & 56,0 & 100,0 & 74,0 & 55,0 & 112,0 & 82,0 \\
\hline $\mathrm{Ca}\left(\mathrm{cmolc} \mathrm{dm}^{-3}\right)$ & 0,6 & 0,4 & 1,4 & 1,5 & 1,3 & 0,6 & 1,0 & 0,8 \\
\hline $\mathrm{Mg}\left(\mathrm{cmolc} \mathrm{dm}^{-3}\right)$ & 0,2 & 0,2 & 0,9 & 1,3 & 1,0 & 0,2 & 0,9 & 0,4 \\
\hline $\mathrm{S}\left(\mathrm{mg} \mathrm{dm}^{-3}\right)$ & 5,9 & 3,7 & 36,1 & 5,1 & 16,0 & 30,7 & 4,4 & 13,2 \\
\hline $\mathrm{SB}\left(\mathrm{cmolc} \mathrm{dm}^{-3}\right)$ & 0,9 & 0,6 & 2,4 & 3,0 & 2,5 & 0,9 & 2,2 & 1,3 \\
\hline $\mathrm{Zn}\left(\mathrm{mg} \mathrm{dm}^{-3}\right)$ & 3,0 & 1,5 & 6,4 & 2,1 & 3,3 & 7,0 & 2,3 & 3,6 \\
\hline $\mathrm{Fe}\left(\mathrm{mg} \mathrm{dm}^{-3}\right)$ & 37,1 & 76,4 & 88,7 & 126,6 & 96,2 & 74,3 & 104,7 & 81,5 \\
\hline $\operatorname{Mn}\left(\mathrm{mg} \mathrm{dm}^{-3}\right)$ & 7,4 & 2,7 & 2,9 & 6,0 & 4,6 & 3,3 & 6,9 & 4,7 \\
\hline $\mathrm{Cu}\left(\mathrm{mg} \mathrm{dm}^{-3}\right)$ & 0,4 & 0,5 & 1,2 & 0,5 & 0,7 & 1,2 & 0,5 & 0,8 \\
\hline$B\left(m g d^{-3}\right)$ & 0,2 & 0,1 & 0,4 & 0,3 & 0,5 & 0,3 & 0,4 & 0,3 \\
\hline $\mathrm{Al}\left(\mathrm{cmolc} \mathrm{dm}^{-3}\right)$ & 0,9 & 0,7 & 0,1 & 0,1 & 0,1 & 0,5 & 0,2 & 0,4 \\
\hline$V(\%)$ & 18,0 & 16,7 & 53,5 & 66,4 & 58,9 & 23,4 & 50,3 & 33,3 \\
\hline m (\%) & 50,8 & 52,0 & 4,0 & 3,3 & 4,0 & 36,3 & 9,5 & 22,5 \\
\hline $\mathrm{H}+\mathrm{Al}\left(\mathrm{cmolc} \mathrm{dm}^{-3}\right)$ & 4,0 & 3,2 & 2,1 & 1,5 & 1,8 & 2,9 & 2,1 & 2,6 \\
\hline $\mathrm{t}\left(\mathrm{cmolc} \mathrm{dm}^{-3}\right)$ & 1,8 & 1,3 & 2,5 & 3,1 & 2,6 & 1,4 & 2,4 & 1,7 \\
\hline $\mathrm{T}\left(\mathrm{cmolc} \mathrm{dm}^{-3}\right)$ & 4,9 & 3,8 & 4,5 & 4,5 & 4,3 & 3,8 & 4,3 & 3,9 \\
\hline $\mathrm{MO}(\%)$ & 3,6 & 1,6 & 1,3 & 1,9 & 1,6 & 1,5 & 2,0 & 1,8 \\
\hline P-rem $\left(\mathrm{mg} \mathrm{dm}^{-3}\right)$ & 34,6 & 32,0 & 34,0 & 33,4 & 32,7 & 32,5 & 31,4 & 30,9 \\
\hline Ca:Mg & 3,0 & 2,0 & 1,6 & 1,2 & 1,3 & 3,0 & 1,1 & 2,0 \\
\hline Areia (\%) & 57 & 56 & & & & & & \\
\hline Silte (\%) & 35 & 19 & & & & & & \\
\hline Argila (\%) & 8 & 25 & & & & & & \\
\hline
\end{tabular}

econômico. Esse fato pode ser evidenciado pela correlação positiva encontrada entre MSR e o índice $m \%\left(r=0,70^{*}\right)$ e $\mathrm{H}+\mathrm{Al}(\mathrm{r}=0,69 *)$. Houve correlação inversa entre MSR e os teores de $\mathrm{Mg}$ disponíveis no solo $\left(\mathrm{r}=-0,77^{*}\right)$. O Ca é um elemento com função chave na proteção das raízes contra os efeitos negativos do pH baixo (Marschner, 1995) e uma inibição da absorção de $\mathrm{Ca}$, devido a maior disponibilidade de $\mathrm{Mg}$ (Mengel \& Kirkby, 2001), pode ter sido a causa desse fato, corroborado pela correlação encontrada entre MSR e $\mathrm{Ca}: \mathrm{Mg}\left(\mathrm{r}=0,85^{*}\right)$.

Considerando as produções de MSPA e MSR (Tabela 3), o tratamento com adubação orgânica promoveu o menor crescimento da arnica, tanto na presença quanto na ausência da calagem. A análise mostrou que o esterco de curral utilizado apresentava baixo teor de P (Tabela 2). Assim, a dose do adubo orgânico usada nesse tratamento (Tabela 2) forneceu quantidade

Tabela 2. Teores totais e quantidades aplicadas de nutrientes presentes no esterco de curral usado para adubação orgânica. Lavras, UFLA, 2004.

\begin{tabular}{lccc}
\hline Macronutrientes & $\mathbf{( \mathbf { g ~ k g } ^ { - 1 } )}$ & Micronutrientes & $\mathbf{~ ( m g ~ k g ~}^{-1} \mathbf{)}$ \\
\hline $\mathrm{N}$ & $16,70(268,9)^{\star}$ & $\mathrm{Mn}$ & $355,82(5,7)^{\boldsymbol{*}}$ \\
$\mathrm{P}$ & $2,65(42,7)$ & $\mathrm{Fe}$ & $9802,55(157,8)$ \\
$\mathrm{K}$ & $10,08(162,3)$ & $\mathrm{Zn}$ & $53,86(0,9)$ \\
$\mathrm{Ca}$ & $6,96(112,1)$ & $\mathrm{Cu}$ & $19,47(31,3)$ \\
$\mathrm{Mg}$ & $5,40(86,9)$ & & \\
$\mathrm{S}$ & $2,29(36,9)$ & & \\
\hline
\end{tabular}

*Valores entre parênteses representam a quantidade de nutrientes, em $\mathrm{mg} \mathrm{dm}^{-3}$, aplicada nos tratamentos com adubação puramente orgânica.

insuficiente deste nutriente, limitando o crescimento da arnica, sugerido pelos valores de correlação encontrados entre o P disponível e MSPA ( $\left.\mathrm{r}=0,69^{*}\right)$ e $\operatorname{MSR}(r=0,69 *)$. Portanto, para a dose de esterco utilizada, a complementação com nutrientes na forma mineral foi essencial para um crescimento adequado das plantas. Corrêa Júnior (1994) afirma que, em muitos casos, a complementação da adubação orgânica, via adubos minerais, é fundamental quando se visa à produção adequada de plantas medicinais. No presente trabalho, o tratamento de adubação mista comprova esse fato, embora a dose mineral ainda tenha sido insuficiente (Tabela 3$)$.

Analisando a produção de MSPA e MSR (Tabela 3) e a composição química do solo controle (Tabela 1), pode-se inferir que a arnica é uma espécie adaptada a altos teores e saturações por Al. Assim, nas condições deste estudo, a 
Tabela 3. Produção de matérias secas de parte aérea e raiz e crescimento relativo das plantas de arnica em função da calagem e adubação e no controle. Lavras, UFLA, 2004.

\begin{tabular}{|c|c|c|}
\hline \multirow{2}{*}{ Adubação } & \multicolumn{2}{|c|}{ Calagem } \\
\hline & Com & Sem \\
\hline & \multicolumn{2}{|c|}{ Matéria seca de parte aérea (g planta-1) } \\
\hline Mineral & 7,64 a $A$ & 7,91 a $A$ \\
\hline Orgânica & $3,26 \mathrm{c} \mathrm{A}$ & 1,97 с B \\
\hline Mista & $6,18 \mathrm{~b} \mathrm{~A}$ & 5,28 b B \\
\hline Controle & & \\
\hline \multirow[t]{2}{*}{$\mathrm{CV}(\%)$} & & \\
\hline & \multicolumn{2}{|c|}{ Matéria seca de raízes (g planta-1) } \\
\hline Mineral & 2,31 a B & 3,09 a $A$ \\
\hline Orgânica & $1,31 \mathrm{~b} \mathrm{~A}$ & $1,14 \mathrm{c} \mathrm{A}$ \\
\hline Mista & 2,39 a $A$ & $2,21 \mathrm{~b} \mathrm{~A}$ \\
\hline Controle & \multicolumn{2}{|c|}{2,91} \\
\hline \multirow[t]{2}{*}{$\mathrm{CV}(\%)$} & \multicolumn{2}{|c|}{14,17} \\
\hline & \multicolumn{2}{|c|}{ Crescimento relativo (\%) } \\
\hline Mineral & 278,01 a $A$ & 311,53 a $A$ \\
\hline Orgânica & 221,31 b A & 118,24 b B \\
\hline Mista & $179,50 \mathrm{~b} \mathrm{~A}$ & $180,82 \mathrm{~b} \mathrm{~A}$ \\
\hline Controle & \multicolumn{2}{|c|}{184,19} \\
\hline $\mathrm{CV}(\%)$ & \multicolumn{2}{|c|}{34,22} \\
\hline
\end{tabular}

Médias seguidas de letras iguais, minúsculas nas colunas e maiúsculas nas linhas, não diferem entre si (Scott-Knott, 5\%).

calagem mostrou ser desnecessária para o crescimento da arnica. Esses resultados estão de acordo com o exposto em Goodland \& Ferri (1979), sobre o crescimento das plantas nativas do Cerrado. Esses autores afirmaram que a tolerância dessas plantas às condições adversas está intimamente relacionada à alta saturação por $\mathrm{Al}(\mathrm{m} \%)$, ou seja, a tolerância aumenta com os valores de $\mathrm{m} \%$ dos solos. Contudo, para explora- ção comercial da arnica, ou qualquer outra atividade que objetive maior crescimento dessa espécie, estudos mais detalhados de nutrição mineral são necessários.

Para o crescimento relativo (CR), os tratamentos com adubação mineral resultaram em incrementos maiores (Tabela 3), provavelmente, porque a pronta disponibilidade de nutrientes foi suficiente para alavancar o crescimento das plantas. Sugere-se que a diferença observada entre os tratamentos orgânicos, com e sem calagem, provavelmente se deveu a uma maior disponibilidade de $\mathrm{S}_{-} \mathrm{SO}_{4}$ no tratamento que recebeu calcário (Tabela 1).

$\mathrm{O}$ acúmulo de determinado nutriente pela planta é função do seu teor no tecido e, principalmente, da produção de massa seca. Assim, observa-se que o acúmulo dos elementos (Tabela 4) seguiu a tendência observada para a massa seca (Tabela 3). Portanto, decresceu na ordem de adubação mineral, mista e orgânica. Nesse último tratamento, destaca-se o baixo acúmulo de $\mathrm{P}$ e $\mathrm{S}$, reflexo dos baixos crescimento (Tabela 3) e teores na planta (Tabela 4).

De maneira geral, os teores dos nutrientes na parte aérea da arnica (Tabela 4) não sofreram variações acentuadas com a aplicação dos tratamentos. Não se encontrou na literatura um referencial nutricional para a arnica. Dessa forma, para efeito de comparação, adotou-se como referência os valores médios citados por Marschner (1995). De maneira geral, os nutrientes N, P e K mantiveram-se abaixo, enquanto que o $\mathrm{Ca}, \mathrm{Mg}, \mathrm{S}, \mathrm{Cu}$ e $\mathrm{Fe}$ situaram-se dentro dos limites citados. Somente os teores de Zn e Mn apresentaram-se acima dos limites, sobretudo no controle, indicando maior exigência da arnica nestes micronutrientes. Os teores de Al também não sofreram variações acentuadas em função dos tratamentos, à exceção da adubação orgânica sem calagem. Neste tratamento, à

Tabela 4. Teores e acúmulos de nutrientes e alumínio na massa seca da parte aérea das plantas de arnica, aos 150 dias de cultivo, em função da calagem e adubação e no controle. Lavras, UFLA, 2004.

\begin{tabular}{|c|c|c|c|c|c|c|c|c|c|c|c|c|c|c|}
\hline \multirow{4}{*}{$\begin{array}{l}\text { Elemento } \\
\mathrm{N}\left(\mathrm{g} \mathrm{kg}^{-1}\right)\end{array}$} & \multicolumn{12}{|c|}{ Adubação } & \multirow{3}{*}{\multicolumn{2}{|c|}{ Controle }} \\
\hline & \multicolumn{2}{|c|}{ Mineral } & \multicolumn{2}{|c|}{ Orgânica } & \multicolumn{2}{|c|}{ Mista } & \multicolumn{2}{|c|}{ Mineral } & \multicolumn{2}{|c|}{ Orgânica } & \multicolumn{2}{|c|}{ Mista } & & \\
\hline & \multicolumn{6}{|c|}{ Com calagem } & \multicolumn{6}{|c|}{ Sem calagem } & & \\
\hline & 13,4 & $(102,1)^{*}$ & 9,1 & $(29,9)$ & 13,0 & $(80,3)$ & 16,8 & $(132,9)$ & 8,0 & $(15,7)$ & 11,5 & $(61,8)$ & 9,7 & $(65,1)$ \\
\hline$P\left(g_{k g}^{-1}\right)$ & 1,4 & $(10,8)$ & 0,8 & $(2,4)$ & 1,4 & $(8,8)$ & 1,3 & $(10,3)$ & 1,0 & $(1,9)$ & 1,5 & $(7,8)$ & 0,6 & $(3,8)$ \\
\hline $\mathrm{K}\left(\mathrm{g} \mathrm{kg}^{-1}\right)$ & 8,8 & $(67,0)$ & 9,6 & $(31,5)$ & 9,6 & $(59,8)$ & 10,5 & $(83,8)$ & 10,7 & $(21,1)$ & 11,2 & $(59,7)$ & 6,8 & $(45,2)$ \\
\hline $\mathrm{Ca}\left(\mathrm{g} \mathrm{kg}^{-1}\right)$ & 8,5 & $(64,8)$ & 6,4 & $(20,6)$ & 6,9 & $(42,4)$ & 6,5 & $(51,2)$ & 7,3 & $(14,4)$ & 5,3 & $(27,4)$ & 8,9 & $(59,7)$ \\
\hline $\mathrm{Mg}\left(\mathrm{g} \mathrm{kg}^{-1}\right)$ & 4,5 & $(34,4)$ & 4,6 & $(14,8)$ & 4,6 & $(28,3)$ & 2,7 & $(21,3)$ & 5,6 & $(11,1)$ & 4,3 & $(22,4)$ & 3,5 & $(23,5)$ \\
\hline $\mathrm{S}\left(\mathrm{g} \mathrm{kg}^{-1}\right)$ & 3,1 & $(23,9)$ & 1,5 & $(5,0)$ & 2,5 & $(15,4)$ & 2,4 & $(18,9)$ & 1,5 & $(2,9)$ & 1,8 & $(9,6)$ & 1,4 & $(9,0)$ \\
\hline $\mathrm{Cu}\left(\mathrm{mg} \mathrm{kg}^{-1}\right)$ & 6,5 & $(0,1)$ & 8,8 & $(0,0)$ & 8,3 & $(0,1)$ & 7,0 & $(0,1)$ & 9,6 & $(0,0)$ & 10,7 & $(0,1)$ & 7,5 & $(0,1)$ \\
\hline $\mathrm{Fe}\left(\mathrm{mg} \mathrm{kg}^{-1}\right)$ & 180,2 & $(1,4)$ & 191,1 & $(0,1)$ & 222,7 & $(1,4)$ & 156,1 & $(1,2)$ & 138,5 & $(0,3)$ & 212,4 & $(1,1)$ & 151,9 & $(1,0)$ \\
\hline $\mathrm{Zn}\left(\mathrm{mg} \mathrm{kg}^{-1}\right)$ & 167,1 & $(1,3)$ & 81,5 & $(0,3)$ & 89,9 & $(0,6)$ & 452,2 & $(3,6)$ & 94,6 & $(0,2)$ & 155,8 & $(0,8)$ & 221,9 & $(1,5)$ \\
\hline $\mathrm{Mn}\left(\mathrm{mg} \mathrm{kg}^{-1}\right)$ & 105,9 & $(0,8)$ & 80,9 & $(0,3)$ & 68,8 & $(0,4)$ & 489,2 & $(3,9)$ & 207,1 & $(0,4)$ & 304,8 & $(1,6)$ & 972,1 & $(6,5)$ \\
\hline $\mathrm{Al}\left(\mathrm{g} \mathrm{kg}^{-1}\right)$ & 65,8 & $(0,5)$ & 71,8 & $(0,2)$ & 78,5 & $(0,5)$ & 71,2 & $(0,6)$ & 58,3 & $(0,1)$ & 91,6 & $(0,5)$ & 56,5 & $(0,4)$ \\
\hline
\end{tabular}

*Valores entre parênteses correspondem aos acúmulos de elementos, em mg planta ${ }^{-1}$ 
semelhança do controle, o menor teor de Al na parte aérea se deve, provavelmente, a indisponibilização através de sua complexação com a matéria orgânica (Andrade et al., 2002).

Todavia, embora tenham sido detectadas algumas variações nos teores dos nutrientes em função dos tratamentos, não foi observado, durante o período de experimento, nenhum sintoma típico de deficiência ou toxidez, inclusive para o Al. Pela classificação de Goodland \& Ferri (1979), a arnica se enquadraria no grupo das plantas tolerantes e não acumuladoras de $\mathrm{Al}$, uma vez que os teores deste elemento situaram-se entre 20 e $200 \mathrm{mg} \mathrm{kg}^{-1}$ de $\mathrm{Al}$ (base massa seca). Os mecanismos que lhe conferem tal tolerância, segundo Mengel \& Kirkby (2001), são de ordem genética e estão em consonância com Goodland \& Ferri (1979) a respeito da adaptação das plantas às condições de solos de Cerrado.

Os campos rupestres, pelas suas condições intrínsecas, são considerados como áreas de preservação permanente (artigo segundo do Código Florestal Lei 4771/65, alterada pela Lei 7803/89). Assim, os teores dos nutrientes e alumínio do tratamento controle não devem ser considerados como um referencial nutricional para comparação com plantas obtidas em condições de cultivo. Para essas condições, o presente trabalho indica como referencial nutricional os teores obtidos no tratamento mineral sem calagem, pois este apresentou maior crescimento e, nos tratamentos minerais a calagem mostrou-se prática desnecessária.

O crescimento (MSPA, MSR e CR) da arnica foi maior com aplicação de fertilizante menor com adubo orgânico. Para MSPA e CR, a calagem foi essencial somente nos tratamentos que receberam adubo orgânico. A arnica não é planta exigente em macronutrientes, mas é em Zn e Mn. Embora não acumuladora, é tolerante ao $\mathrm{Al}$ do solo.

\section{REFERÊNCIAS}

ALVAREZ VVH; NOVAIS RF; DIAS LE; OLIVEIRA JA. 2000. Determinação e uso do fósforo remanescente. Boletim Informativo da Sociedade Brasileira de Ciência do Solo 25: 27-32.

ANDRADE AT; FERNANDES LA; FAQUIN V. 2002. Organic residue, limestone, gypsum, and phosphorus adsorption by lowland soils. Scientia Agricola 59: 349-355.

CARVALHO DA. 1992. Flora fanerogâmica de campos rupestres da Serra da Bocaina, Minas Gerais: caracterização e lista de espécies. $\mathrm{Ci}$ ência e Prática 16: 97-122.

COMISSÃO DE FERTILIDADE DO SOLO DO ESTADO DE MINAS GERAIS. 1999. Adubação Orgânica. In: Ribeiro AC; Guimarães PTG; Alvarez VVH (eds). Recomendações para o uso de corretivos e fertilizantes em Minas Gerais. Viçosa: CFSEMG. p. 87-92.

CORRÊA JÚNIOR C. 1994. Influência das adubações orgânica e química na produção de camomila [Chamomilla recutita (L.) Raucschert] e do seu óleo essencial. Jaboticabal: UNESP. 96p. (Tese mestrado).

EMPRESA BRASILEIRA DE PESQUISA AGROPECUÁRIA.1997. Serviço Nacional de Levantamento e Conservação de Solo. Manual de métodos de análise de solo. Rio de Janeiro: Ministério da Agricultura. 212p.

GOODLAND RJA; FERRI MG. 1979. Ecologia do cerrado. São Paulo: Ed. da Universidade de São Paulo. 193p. (Reconquista do Brasil, v. 52).

MALAVOLTA E; VITTI GC; OLIVEIRA SA. 1997. Avaliação do estado nutricional das plantas-princípios e aplicações. Piracicaba: POTAFOS. 210p.
MARSCHNER H. 1995. Mineral Nutrition of higher plants. Londres: Academic Press. 889p.

MENGEL K; KIRKBY EA. 2001. Principles of plant nutrition. 5.ed. Netherlands: Kluwer Academic Publishers. 849p.

NOVAIS RF; NEVES JCL; BARROS NF. 1991. Ensaio em ambiente controlado. In: OLIVEIRA AJ; GARRIDO WE; ARAÚJO JD; LOURENÇO S (coord). Métodos de pesquisa em fertilidade do solo. Brasília: Embrapa-SEA. p. 189-253 (Embrapa-SEA Documentos, 3)

OLIVEIRA-FILHO AT; FLUMINHAN-FILHO M. 1999. Ecologia da vegetação do Parque Florestal Quedas do Rio Bonito. Cerne 5: 5164.

OLIVEIRA JÚNIOR AC; FAQUIN V; PINTO JEBP; LIMA SOBRINHO RR; BERTOLUCCI SKV. 2005. Teor e rendimento de óleo essencial no peso fresco de arnica, em função de calagem e adubação. Horticultura Brasileira 23: 735-739.

PARON ME; MING LC; HABER LL; ALMEIDA CIM; MING LC. 2005. Conservação de espécies de Lychnophora: sistema de informações sobre procedimentos. Horticultura Brasileira 23: Suplemento. CD-ROM.

PINHEIRO RC. 2002. Abordagem fitoquímica, rendimento do óleo essencial de Lychnophora pinaster Mart. utilizando dois métodos de secagem. Lavras: UFLA. 41p. (Tese mestrado).

SEMIR J. 1991. Revisão taxonômica de Lychnophora Mart. (Veroniaceae: Compositae). Campinas: UNICAMP. 515p. (Tese doutorado).

SILVA SMP. 1994. Aspectos da fenologia e da reprodução sexuada da arnica (Lychnophora pinaster Mart.)-Asteraceae. Lavras: UFLA. 45p. (Tese mestrado).

SOCIEDADE BOTÂNICA DO BRASIL. 1992. Centuria plantarum brasiliensium extitions miniata. Brasília. 167p.

SOUZA AV. 2003. Propagação in vitro e aspectos anatômicos de arnica (Lychnophora pinaster) Mart. 2003. Lavras: UFLA. 127p. (Tese mestrado).

TEDESCO MJ; GIANELO C; BISSANI CA; BOHEN H; VOLKWEIS SJ. 1995. Análise de solo, planta e outros materiais. Porto Alegre: UFRGS. 174p. 